\title{
The Efficacy of Dignity Therapy on the Psychological Well-Being in Loved Ones of Terminally III Patients
}

\author{
Miguel Julião, MD, MSc, PhD
}

\section{Dear Editor:}

Dignity therapy (DT) is a novel, brief, individualized intervention, which gives terminally ill patients the opportunity to convey memories and important disclosures and to prepare a legacy document that can be given to patients for them to share or bequeath to individuals of their choice. A previously published DT study showed that family members found DT helpful in terms of enhanced patient dignity (78\%), $72 \%$ reported that it heightened meaning of life for the patient (72\%), the document produced from DT was a comfort to them in their time of grief (78\%), and most would recommend DT for other patients and families (95\%). ${ }^{1}$

In view of these data, we recently conducted a study that randomized patients to either DT and standard palliative care (SPC) or SPC alone to evaluate DT's effect on psychological well-being of both terminally ill patients ${ }^{2,3}$ and their loved ones (family members, friends, other significant, and close caregivers).

In this letter, we present data evaluating the effects of DT on the loved ones of terminally ill patients. In this study, psychological well-being was assessed using the Mental Health Inventory (Mental Health Index [MHI]: minimum 38; maximum 226) at baseline and four days after the intervention. Of the 80 terminally ill patients enrolled, $45(56 \%)$ had someone in their lives they wished to bequeath a legacy document to. Of these 45 patients, 25 (55\%) agreed to participate in the study and provided written informed consent; $52 \%$ were male. In these 25 participants, 15 were randomized to DT and 10 to the SPC group. Overall baseline mean MHI in the loved ones was 131 (range: 38-226), indicating a moderate-to-high level of psychological well-being. Legacy documents were given to family members in $67 \%$ of the cases, $30 \%$ of the terminally ill patients did not want to deliver the legacy document, and 3\% of patients gave it to friends. The results showed no significant difference between baseline and post-intervention MHI scores within each study group (Table 1). Similarly, no significant differences between subjects in the two study arms were observed in MHI between baseline and post-intervention (baseline: mean's difference $=$ $1.54 ; 95 \% \mathrm{CI}[-24.43$ to 27.51$] ; p=0.9041$; post-intervention: mean's difference $=-12.68 ; 95 \%$ CI $[-43.52$ to 18.16$]$; $p=0.4046$ ) .

Although this study appeared to show no effect of DT, there are important study limitations. First, the study was small because of the low percentage of patients who had a loved one they wanted to leave legacy documents to. Second, the fact that only $55 \%$ of these loved ones agreed to participate. Another factor is the high baseline MHI scores in both the DT and SPC groups. These scores would suggest that these loved ones already had a moderate-to-high level of psychological well-being and it would be challenging for an intervention to show a further increase or effect.

This study has tried to answer some of the unanswered questions still remaining about DT's effect on caregivers' well-being. As ultimately although DT did not improve the psychological well-being of loved ones in our study-likely because of the high baseline MHI scores-DT may ultimately prove itself to be an important tool within the setting of any multidisciplinary palliative care setting. Thus, even though this study is "negative," we realized that it is important to publish these data so that future researchers in this area can learn from our experience.

\section{Acknowledgments}

The author would like to thank Dr. Mark Clemons for his ideas and help revising this article. A special word of gratitude to Professor Harvey Chochinov for his constant support, wisdom, and friendship. This research received no specific funding.

Table 1. Comparison of Mental Health Index Scores for Each of the Intervention Groups

\begin{tabular}{lcccc}
\hline Study arm & Baseline & Post-intervention & Difference & $\mathrm{p}$ \\
\hline DT (mean, SD) & $136.79(30.05)$ & $132.57(30.95)$ & $4.21 ; 95 \%$ CI [-6.29 to 14.72] & 0.4018 \\
SPC (mean, SD) & $142.90(42.77)$ & $145.18(47.08)$ & $-2.27 ; 95 \%$ CI [-7.79 to 3.25] & 0.3809 \\
\hline
\end{tabular}

CI, confidence interval; DT, dignity therapy; SD, standard deviation; SPC, standard palliative care.

Escola de Medicina da Universidade do Minho, Braga, Portugal. 


\section{References}

1. McClement S, Chochinov HM, Hack T, et al.: Dignity therapy: Family member perspectives. J Palliat Med 2007; 10:1076-1082.

2. Julião M, Oliveira F, Nunes B, et al.: Efficacy of dignity therapy on depression and anxiety in Portuguese terminally ill patients: A phase II randomized controlled trial. J Palliat Med 2014;17:688-695.

3. Julião M, Oliveira F, Nunes B, et al.: Effect of dignity therapy on end-of-life psychological distress in terminally ill
Portuguese patients: A randomized controlled trial. Palliat Support Care 2017;7:1-10.

Address correspondence to:

Miguel Julião, MD, MSc, PhD

Escola de Medicina da Universidade do Minho

Campus de Gualtar

Braga 4710-057

Portugal

E-mail: migueljuliao@gmail.com 